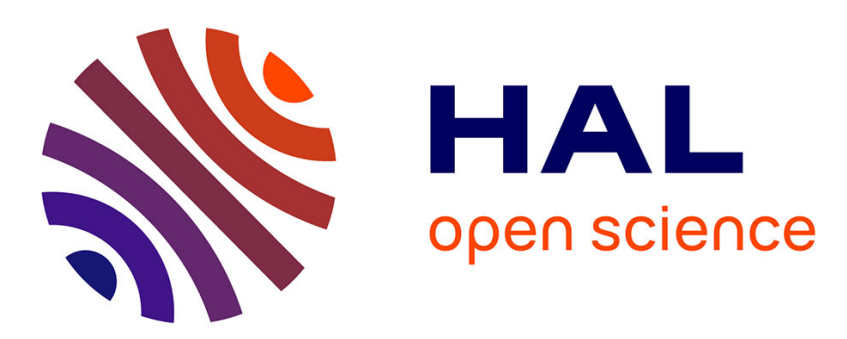

\title{
L'intégration territoriale des gares sur lignes à grande vitesse en France: une approche typologique
}

Valérie Facchinetti-Mannone, Cyprien Richer

\section{To cite this version:}

Valérie Facchinetti-Mannone, Cyprien Richer. L'intégration territoriale des gares sur lignes à grande vitesse en France: une approche typologique. RTS - Recherche Transports Sécurité, 2011, 27 (3), pp.200-214. 10.1007/s13547-011-0014-4 . halshs-00608751

\section{HAL Id: halshs-00608751 \\ https://shs.hal.science/halshs-00608751}

Submitted on 9 Aug 2011

HAL is a multi-disciplinary open access archive for the deposit and dissemination of scientific research documents, whether they are published or not. The documents may come from teaching and research institutions in France or abroad, or from public or private research centers.
L'archive ouverte pluridisciplinaire HAL, est destinée au dépôt et à la diffusion de documents scientifiques de niveau recherche, publiés ou non, émanant des établissements d'enseignement et de recherche français ou étrangers, des laboratoires publics ou privés. 


\title{
L'intégration territoriale des gares sur lignes à grande vitesse en France : une approche typologique
}

\author{
Valérie Facchinetti-Mannone
}

Laboratoire ThéMA, UMR 6049 CNRS, Universités de Franche-Comté et de Bourgogne valerie.mannone@u-bourgogne.fr

et

\section{Cyprien Richer}

CETE Nord-Picardie,

Ministère de l'Ecologie, du Développement durable, des Transports et du Logement,

Pôle de Compétences et d'Innovation CERTU « Transports du Quotidien »

cyprien.richer@developpement-durable.gouv.fr

\section{Résumé}

La construction de Lignes à Grande Vitesse (LGV) en France et en Europe a bouleversé l'accessibilité ferroviaire des territoires «branchés " à ce réseau de transport rapide. Les gares nouvelles situées sur les LGV tirent l'avantage maximum de ces gains relationnels. Cependant, l'accès en transport collectif à ces gares pose souvent problème. En effet, leur localisation, souvent ex-urbanisée, n'a pas été dictée par un souci d'offrir une desserte performante par les réseaux de transport en commun, notamment ferroviaire. Cette situation s'avère problématique pour le pré et post-acheminement des voyageurs, et oblige les acteurs locaux à développer des dessertes nouvelles de qualité variable. Cet article propose une analyse de la qualité de l'intégration territoriale des gares TGV par une typologie des interactions entre ces nouveaux points de desserte et les gares historiques, en position centrale. Dans un contexte marqué par la mise à contribution croissante des collectivités locales au financement des nouvelles infrastructures ferroviaires de la grande vitesse, nous examinerons si cette évolution des jeux d'acteurs encourage une meilleure prise en compte du positionnement des gares TGV par rapport à des critères d'accessibilité en transport collectif.

\section{Mots-clés}

Grande vitesse ferroviaire, accessibilité, système de transport, gare ferroviaire, aménagement 


\section{Introduction}

En France, l'extension du réseau de lignes à grande vitesse ferroviaire vise à relier le plus directement et le plus rapidement possible les grandes métropoles. Si ces dernières sont desservies en leur centre, par l'intermédiaire de leur gare historique et/ou d'une nouvelle gare, la plupart des autres aires urbaines du territoire, écartées du tracé des LGV, sont connectées au réseau à grande vitesse par l'intermédiaire des lignes classiques ou de gares nouvelles construites sur les LGV. Ces "gares TGV " ${ }^{1}$, souvent ex-urbanisées, imposent un dédoublement de la fonction ferroviaire susceptible de bouleverser l'organisation et les dynamiques spatiales des aires urbaines desservies. Elles posent notamment la question du devenir des gares historiques, ferment de l'urbanisation au siècle dernier.

Le doublement de la desserte ferroviaire suscite de nombreuses questions relatives aux conséquences du partage de la desserte sur la fréquentation et le fonctionnement des gares. Comment s'effectuent les relations entre les deux gares de l'aire urbaine ? Quelles sont les conséquences de ce dédoublement de la fonction ferroviaire sur la répartition de la desserte et plus largement sur l'organisation des aires urbaines? Autant d'interrogations qui se posent aujourd'hui avec une nouvelle acuité dans le cadre des projets de nouvelles lignes à grande vitesse. La question des relations entre gare TGV et gare historique, autrefois secondaire, est en effet aujourd'hui au cœur des réflexions que mènent opérateurs ferroviaires et collectivités locales autour de l'intégration territoriale des nouvelles gares périphériques. Cette évolution nous conduit à interroger les facteurs de "l'intégration territoriale " des gares TGV, c'est à dire leur assimilation aux réseaux de transport et de mobilité et potentiellement aux dynamiques urbaines de l'aire urbaine desservie.

Afin d'analyser l'interdépendance du système de desserte liée à la coexistence de deux gares ferroviaires, l'approche retenue propose d'observer la diversité des configurations territoriales liées à ces nouvelles implantations ferroviaires par une analyse typologique multicritère en trois temps :

- Dans un premier temps, nous revisitons la localisation des gares dans les aires urbaines pour montrer la diversité des configurations spatiales, premier critère potentiel d'intégration territoriale des gares TGV.

- Ce premier cadrage permet, dans un deuxième temps, d'observer comment la situation des gares au sein de l'aire urbaine et du réseau ferroviaire détermine le partage fonctionnel qui s'établit entre gare TGV et gare historique et comment ce dédoublement se traduit en termes de desserte et de fréquentation des gares. L'approche typologique retenue se fonde sur la combinaison de critères spatiaux relatifs à la position de la nouvelle gare TGV au sein du réseau ferroviaire et de critères fonctionnels permettant de préciser les caractéristiques de la desserte de chaque gare et les spécificités de leur fréquentation respective.

- Le troisième temps s'intéresse plus spécifiquement à la connexion entre les deux platesformes ferroviaires. II s'agit de déterminer par le biais d'une typologie croisant des critères modaux (nature et fréquence de l'offre de rabattement), temporels (temps du trajet en transports collectifs entre les deux gares et temps moyen de correspondance) et des critères relatifs au niveau de service (tarification, signalétique, information multimodale, confort des cheminements ...), le degré de performance des systèmes de relations mis en place entre la gare TGV nouvelle et la gare historique.

La démarche méthodologique s'appuie sur une schématisation des principaux critères utilisés pour déterminer les types de gares dans chacun des trois temps. Le croisement de cette triple approche typologique souligne la grande variété des configurations liées au partage fonctionnel des gares et à la performance des liaisons intergares. L'analyse questionne un système TGV qui peut se trouver, sous certain aspect, fragilisé par une médiocre insertion territoriale des gares.

\footnotetext{
$1 \quad$ Nous employons l'expression «Gare TGV » pour caractériser les gares construites sur Lignes nouvelles à
} Grande Vitesse (LGV). 


\section{$1 /$ Des localisations majoritairement périphériques imposées par le modèle français de la grande vitesse}

\section{1-1/ Principes d'implantation des nouvelles gares TGV}

La France possède le réseau de lignes à grande vitesse le plus ancien (la première LGV relie Paris à Lyon dès le début des années 80 ) et jusqu'en décembre 2010, date de mise en service de la LGV reliant Valence et Albacete qui porte le réseau espagnol à $2056 \mathrm{~km}$, longtemps le plus étendu (1 $896 \mathrm{~km}$ ) d'Europe. Conçu pour pallier la saturation des lignes classiques et concurrencer l'avion, le réseau présente une structure radiale, centrée sur Paris, qui reflète la centralisation du territoire français et les déséquilibres de son armature urbaine. Privilégiant une logique d'efficacité à l'échelle nationale selon les termes de Philippe Menerault (2007), le système à grande vitesse français repose sur la réalisation de nouvelles lignes ferroviaires dont les caractéristiques techniques permettent des relations rapides et directes entre Paris et les principales métropoles régionales et européennes (voir figure $n^{\circ} 1$ ):

- Conçue pour relier Paris à Lyon en 2h00, la LGV Sud-Est (1981) est prolongée vers la vallée du Rhône en 1994 (LGV Rhône-Alpes), puis le littoral méditerranéen en 2001 (LGV Méditerranée) pour desservir Marseille et Montpellier ;

- La LGV Atlantique (1990) a rapproché la capitale des grandes villes de l'Ouest (Rennes, Nantes, Tours, Bordeaux) ;

- La LGV Nord (1993) a développé les liaisons directes de Paris vers Lille, vers les " capitales » européennes (Londres en 1993 et Bruxelles en 1997), et plus récemment vers Cologne et Amsterdam (2009) ;

- La première tranche de la LGV Est, mise en service en 2007, relie Paris à Strasbourg et Francfort.

Complétant cette armature, la LGV « d'interconnexion Est» connecte dès 1994 les radiales à grande vitesse et permet la mise en place de relations interrégionales entre métropoles de province sans rupture de charge dans les gares parisiennes. La morphologie du réseau et la rentabilité de l'exploitation de ces liaisons « intersecteurs » imposent un passage par l'lle-deFrance et la desserte d'une ou plusieurs des gares TGV franciliennes. Enfin, la LGV Rhin-Rhône (dont la première tranche sera mis en service en 2011), présentée comme la "première transversale » du réseau à grande vitesse, a pourtant de fortes allures de nouvelle radiale parisienne vers Mulhouse, Bâle et Zurich (Richer, Bérion, 2010).

La constitution progressive de ce réseau de lignes à grande vitesse s'est accompagnée de la construction ex nihilo de gares sur la nouvelle infrastructure ferroviaire. Ces nouveaux points de desserte TGV, vitrines de la modernité du réseau et de l'accès à la grande vitesse, ont déjà fait l'objet d'approches typologiques multiples (P. Zembri (1992), J. Ollivro (1994), J-F. Troin (1995), V. Facchinetti-Mannone (1999) P. Menerault (2008)), qui ont permis d'améliorer la connaissance des types de localisation des dessertes à grande vitesse et d'affiner progressivement les critères d'implantation des gares TGV. Prolongeant ces contributions, notre article propose une analyse fine des articulations (synergie, indifférence, concurrence?) entre les gares implantées sur le réseau LGV et les gares ferroviaires préexistantes.

Le vocable de "gare TGV », par lequel nous désignons les gares constituant notre échantillon, mérite d'être précisé pour éviter toute confusion. Plusieurs distinctions, d'une valeur inégale, sont en effet possibles:

- la distinction entre gares historiques, réalisées au $19^{\text {ème }}$ ou au début du $20^{\text {ème }}$ siécle, et gares nouvelles, nées de la mise en place du réseau à grande vitesse est discutable. Certaines gares «nouvelles » comme celles du Creusot TGV ou de Mâcon-Loché sont en effet en service depuis une trentaine d'années, et la prise en compte de l'ancienneté des gares risquait d'introduire des confusions avec des gares « nouvelles La Part-Dieu, ouverte en 1983, ou la gare du Futuroscope, mise en service en 2000), non positionnées sur le 
réseau LGV. De plus, la compatibilité des LGV avec le réseau classique permet aux "gares historiques » d'accueillir des TGV.

- La distinction entre gares desservies par TGV et gares non desservies se révèle de son côté peu opératoire, étant donnée la quantité et la diversité des gares accueillant par l'intermédiaire du réseau classique une desserte TGV. D'ailleurs, les plus grandes gares du point de vue de la fréquentation TGV ne sont pas les gares construites sur le réseau à grande vitesse, mais les gares historiques des principales agglomérations. Ainsi, la clientèle TGV de la Gare Lille-Flandres est supérieure à celle de Lille-Europe pourtant première gare sur LGV par sa fréquentation.

- L'opposition entre gare centrale, insérée à proximité du centre de l'agglomération, et gare périphérique reste imprécise. Certaines gares sur LGV sont en position centrale (LilleEurope) ou en bordure de l'espace aggloméré, alors que d'autres, localisées sur le réseau classique, peuvent être considérées comme excentrées: c'est le cas de la gare de StPierre-des-Corps par rapport à Tours, ou de la gare du Futuroscope par rapport à Poitiers.

La distinction par rapport à la position de la gare sur le réseau ferroviaire (LGV ou réseau conventionnel) nous paraît être le choix le plus robuste dans le cadre d'une analyse visant à approfondir les liens complexes entre deux types de plates-formes ferroviaires desservant une même aire urbaine. Notre échantillon de " gares TGV » (cf. fig. 1 et 2) est ainsi constitué de gares implantées sur le réseau ferroviaire à grande vitesse.

Figure 1 : Carte du réseau à grande vitesse français à l'horizon 2012 et des gares nouvelles

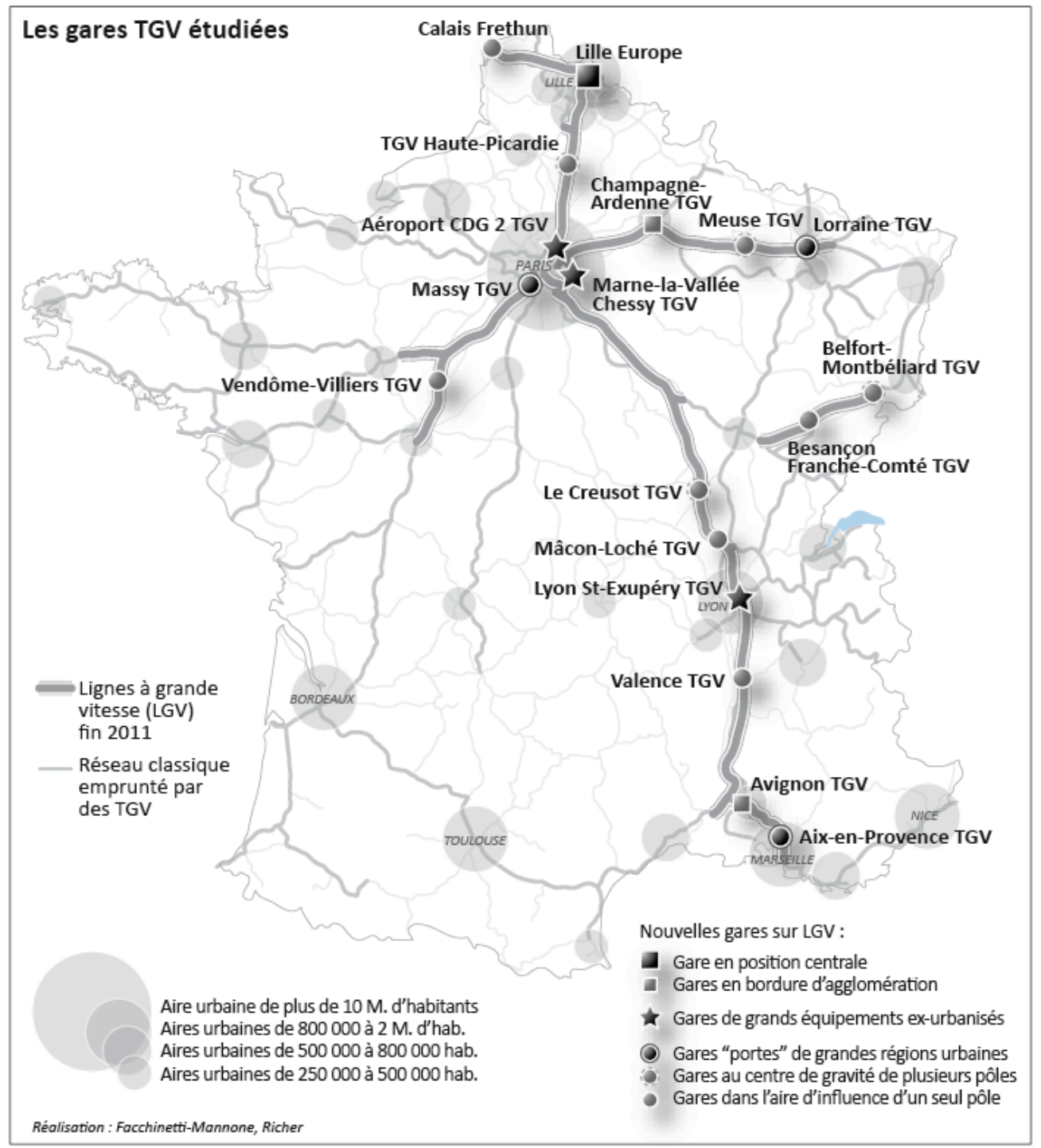


Figure 2: Tableau des 18 gares françaises sur LGV constituant notre échantillon

\begin{tabular}{|c|c|c|c|c|}
\hline Gare TGV nouvelle & Ouverture & $\begin{array}{l}\text { Fréquentation } \\
2008 \text { (SNCF) }\end{array}$ & $\begin{array}{l}\text { Aires urbaines à proximité } \\
\text { (INSEE 1999, rayon de } 40 \text { km) }\end{array}$ & $\begin{array}{l}\text { Population des } \\
\text { aires urbaines } \\
\text { à proximité } \\
\text { (Insee) }\end{array}$ \\
\hline Le Creusot TGV & 1981 & 780000 & $\begin{array}{l}\text { Le Creusot, Montceau-les- } \\
\text { Mines, Autun, Chalon-s-S. }\end{array}$ & 250000 \\
\hline Mâcon-Loché TGV & 1981 & 460000 & Mâcon, Bourg-en-Br. & 190000 \\
\hline Vendôme TGV & 1990 & 355000 & Vendôme, Blois, Chateaudun & 170000 \\
\hline Massy TGV & 1991 & 1400000 & Paris & 11170000 \\
\hline TGV Haute-Picardie & 1994 & 430000 & Amiens, St-Quentin, Péronne & 390000 \\
\hline Lille-Europe & 1994 & 3900000 & Lille & 1140000 \\
\hline Calais-Frethun & 1994 & 200000 & Calais, Boulogne-sur-Mer & 260000 \\
\hline $\begin{array}{l}\text { Aéroport Charles-de- } \\
\text { Gaulle } 2 \text { TGV }\end{array}$ & 1994 & 3400000 & Paris & 11170000 \\
\hline $\begin{array}{l}\text { Marne-la-Vallée } \\
\text { Chessy }\end{array}$ & 1994 & 2600000 & Paris & 11170000 \\
\hline Lyon St-Exupéry TGV & 1994 & 490000 & Lyon & 1650000 \\
\hline Valence TGV & 2001 & 2115000 & $\begin{array}{l}\text { Valence, Roman-s-Is., Livron, } \\
\text { Privas, Tournon, Montélimar }\end{array}$ & 350000 \\
\hline Avignon TGV & 2001 & 2900000 & $\begin{array}{l}\text { Avignon, Orange, Bagnols, } \\
\text { L'Isle-s.S, Cavaillon, Beaucaire }\end{array}$ & 420000 \\
\hline Aix-en-Provence TGV & 2001 & 1750000 & $\begin{array}{l}\text { Marseille-Aix, Salon-de-Pr., } \\
\text { Istres, Miramas, Pertuis, Fos }\end{array}$ & 1620000 \\
\hline $\begin{array}{l}\text { Champagne-Ardenne } \\
\text { TGV }\end{array}$ & 2007 & 545000 & $\begin{array}{l}\text { Reims, Châlons-en-Ch., } \\
\text { Epernay }\end{array}$ & 410000 \\
\hline Lorraine TGV & 2007 & 455000 & $\begin{array}{l}\text { Nancy, Metz, Pont-à-Mousson, } \\
\text { Toul }\end{array}$ & 890000 \\
\hline Meuse TGV & 2007 & 105000 & Verdun, Bar-le-Duc & 68000 \\
\hline Besançon FC TGV & dec. 2011 & $\begin{array}{r}1100000 \\
\text { (prévisions) }\end{array}$ & Besançon, Vesoul & 270000 \\
\hline $\begin{array}{l}\text { Belfort-Montbéliard } \\
\text { TGV }\end{array}$ & dec. 2011 & $\begin{array}{r}1100000 \\
\text { (prévisions) }\end{array}$ & Belfort, Montbéliard, Lure & 300000 \\
\hline
\end{tabular}

Au cœur des enjeux d'accessibilité et d'organisation territoriale, le positionnement de ces « gares TGV " s'inscrit au cœur d'un système multiscalaire dont le fonctionnement tend à une optimisation triple de la vitesse, à l'échelon national, de la desserte, à l'échelon régional, et des potentialités de valorisations locales (Facchinetti-Mannone, Bavoux, 2008 et 2010). Seulement, pour des villes en situation intermédiaire, la subordination des logiques de desserte et de développement territorial à la vitesse, se traduit par l'aménagement de nouvelles gares ex-urbanisées en plus ou moins proche périphérie de l'aire urbaine.

Pour le gestionnaire d'infrastructure (RFF) et l'exploitant du réseau TGV (SNCF), l'implantation de gares en périphérie urbaine permet généralement de résoudre la difficile équation posée par l'arbitrage entre maîtrise des coûts (coûts de construction et d'exploitation) et rentabilité commerciale. Selon les travaux de Laurent Chapelon et Ronan Leclerc (2007), ces nouvelles gares périphériques permettent en effet:

- d'améliorer les temps de parcours (et donc les gains de trafic) entre les grandes villes situées aux extrémités des axes nationaux et internationaux desservis, puisque leur desserte est moins pénalisante pour les temps de trajet: un arrêt sur ligne nouvelle représente une " perte » de 7 à 8 minutes, contre 15 à 20 minutes pour une gare de centre ville ; 
- de faire bénéficier les territoires traversés d'une desserte TGV (minimisant pour ces derniers le risque de "voir passer les TGV ») tout en libérant des sillons sur les lignes classiques, sillons que l'on peut réaffecter aux TER ou au trafic fret ;

- d'élargir l'aire de chalandise des gares, à condition toutefois qu'elles bénéficient d'une accessibilité régionale performante ;

- de réduire les contraintes techniques, spatiales et financières imposées par la densité urbaine à l'intégration des LGV et des gares; tout en résolvant les problèmes posés, dans certains cas, par la capacité insuffisante des lignes classiques ou des gares centrales.

Les gares sur LGV répondent certes aux enjeux de rentabilité économique dictés par l'exploitation du système, mais posent de nombreuses problématiques aux usagers, qui privilégient la commodité d'accès à la grande vitesse, et aux acteurs locaux, soucieux de l'intégration territoriale de ces gares. En effet, ces gares s'inscrivent dans des configurations ferroviaires et spatiales différentes selon les caractéristiques des territoires desservis, et le dédoublement de la desserte qu'elles imposent est susceptible de bouleverser l'organisation et les dynamiques spatiales des aires urbaines concernées.

\section{1-2/ Des gares TGV inscrites dans des configurations territoriales variées}

La plupart de ces nouvelles gares sont localisées en plus ou moins proche périphérie urbaine, à l'exception de la gare de "Lille-Europe » construite sur la LGV Nord en position centrale, qui constitue un cas unique. Bien qu'aménagée spécifiquement lors de l'arrivée du TGV à Lyon, la gare de la Part Dieu se distingue de "Lille Europe » dans la mesure où la gare a été implantée sur les voies ferrées existantes, à l'emplacement d'une ancienne gare de marchandises, se substituant ainsi à la gare historique des Brotteaux située à quelques centaines de mètres et fermée au début des années 1980. Le cas de "Lille Europe » est intégré à notre analyse mais, par son positionnement spécifique, demeure en décalage avec les problématiques d'accessibilité des autres gares construites sur LGV. A Lille, la proximité de la gare historique de Lille-Flandres a favorisé, pour reprendre l'expression d'Alain Barré (2001), la constitution d'un " espace nodal » central, plutôt que la mise en place d'une liaison spécifique entre deux gares aux localisations différentes.

Les autres gares analysées présentent des localisations très diverses par rapport aux aires urbaines qu'elles desservent. Certaines d'entre elles, en périphérie d'aires métropolitaines, ont une localisation dictée par le besoin de desservir de grands équipements ex-urbanisés, générateurs de trafic, du type plates formes aéroportuaires (Charles de Gaulle 2 TGV ou Lyon-St-Exupéry) pour constituer des trinômes d'interconnexion (Varlet, 1992), ou parcs de loisirs (Marne-la-Vallée Chessy).

D'autres répondent plus précisément au vocable de "gares-bis", définies comme des gares nouvelles localisées sur LGV en situation périphérique et dédoublant la desserte ferroviaire d'une aire urbaine. Elles présentent deux types de localisation (voir figure $n^{\circ} 3$ ) :

Certaines sont situées en périphérie d'un pôle unique, en limite du tissu bâti de l'agglomération ou sur un site ex-urbanisé intégré dans l'aire d'influence du pôle urbain, même si comme à Valence ou Avignon, leur aire de chalandise dépasse le périmètre de l'aire urbaine.

- D'autres gares TGV, communes à plusieurs pôles, ont été implantées au centre de gravité de plusieurs aires urbaines petites ou moyennes ou au cœur d'aires métropolitaines. Dans ce dernier cas nous avons choisi d'emprunter à Philippe Menerault $(2007,2008,2010)$ l'expression de "gares portes » de grandes régions urbaines. 
Figure 3 : Typologie de la localisation des gares TGV

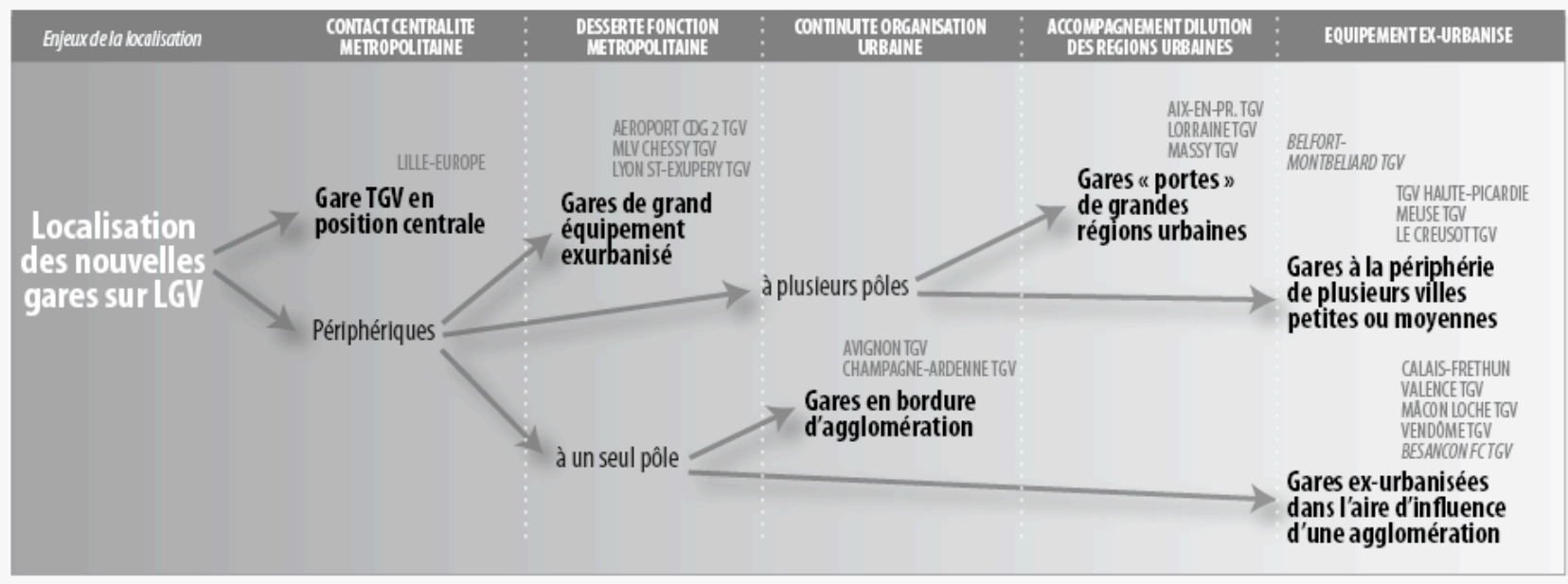

Chacune des gares TGV analysées est couplée avec la ou les gares centrales des aires urbaines desservies. Quelques couples de gares doivent toutefois être justifiés. C'est le cas en particulier, des gares TGV franciliennes " associées » aux grandes gares parisiennes. Ces gares terminus centrales, reliées entre elles par un réseau de transport urbain performant (RER et/ou métro), disposent d'une desserte TGV importante vers les métropoles régionales. Les gares TGV de l'interconnexion francilienne restent, dans ce contexte, des équipements additionnels destinés à offrir aux populations des couronnes métropolitaines un accès complémentaire au réseau à grande vitesse. Dans ce cas, le couplage entre gare périphérique et gare centrale n'a qu'un enjeu modeste, les dessertes des deux types de gares n'étant pas interdépendantes mais dédoublées.

De même, si la gare TGV d'Aix-en-Provence peut-être considérée comme la « gare-bis » de la ville éponyme, son positionnement et son fonctionnement nous amènent plutôt à la considérer comme la «gare porte » de l'aire métropolitaine marseillaise au barycentre de plusieurs centralités et équipements métropolitains (comme l'aéroport de Marseille-Provence entre Marignane et Vitrolles). Elle est en effet davantage le " pendant » de la gare de Marseille St-Charles que de la petite gare régionale d'Aix-en-Provence. Néanmoins, considérer Aix-en-Provence-TGV par rapport à l'agglomération aixoise conserve une pertinence puisque l'enjeu d'accessibilité avec la gare TGV implantée sur le site de l'Arbois demeure très fort. Ajoutons que d'un point de vue institutionnel, ces liens sont également importants puisque la communauté d'agglomération du Pays d'Aix cherche à promouvoir son identité dans une aire métropolitaine morcelée et ainsi développer une bonne accessibilité nationale et internationale (via l'aéroport de Marseille-Provence) qui ne dépendrait pas directement de Marseille.

Précisons également que le cas de Saint Pierre des Corps, considéré comme la gare TGV de Tours, est particulier: comme à Lyon Part Dieu, il s'agit d'un nouveau Bâtiment Voyageurs construit sur le réseau conventionnel à l'emplacement d'un site ferroviaire hérité, qui permet au TGV Atlantique, comme aux trains Paris-Bordeaux avant lui, d'éviter le rebroussement qu'implique la desserte de la gare centrale de Tours en terminus. Pour conserver un échantillon homogène, nous n'intégrons pas ce cas à notre étude même si les problématiques concernant le partage de la desserte ou les relations entre Saint Pierre des Corps et Tours sont assez proches des autres couples de gares.

La création de ces nouvelles gares TGV est à l'origine d'en dédoublement de l'accès au réseau qui impose une redéfinition des dessertes et des fonctions des gares et modifie de façon parfois importante le fonctionnement du système ferroviaire local. 


\section{2/ Le doublement de la desserte ferroviaire: de l'émergence de systèmes ferroviaires métropolitains à la juxtaposition de nœuds d'échelles différentes}

La situation des nouvelles gares au sein des aires urbaines influence le partage fonctionnel qui s'établit entre gare TGV et gare historique à la suite de la mise en service d'une LGV. Cette nouvelle distribution de la desserte entre les deux plates formes ferroviaires s'inscrit dans des configurations variées selon la taille des villes desservies, la situation de la gare et l'existence ou non d'une connexion entre réseau classique et LGV (Facchinetti-Mannone, Richer, 2008). L'impact du dédoublement de la fonction ferroviaire a été en outre plus ou moins marqué selon la position et la fonction qu'occupait la gare historique au sein du réseau classique. Une analyse typologique des couples de gares en fonction des caractéristiques de leur desserte (fréquences de dessertes, types de liaisons et diversité des destinations) et de l'incidence qu'a eue la grande vitesse sur leur fréquentation respective permet de distinguer 4 grands enjeux de partage de la desserte ferroviaire (voir figure $n^{\circ} 4$ ) et de préciser ce lien entre localisation de la gare et partage fonctionnel.

Figure 4 : Typologie du partage de la desserte grandes lignes entre gare sur LGV et gare historique sur réseau classique

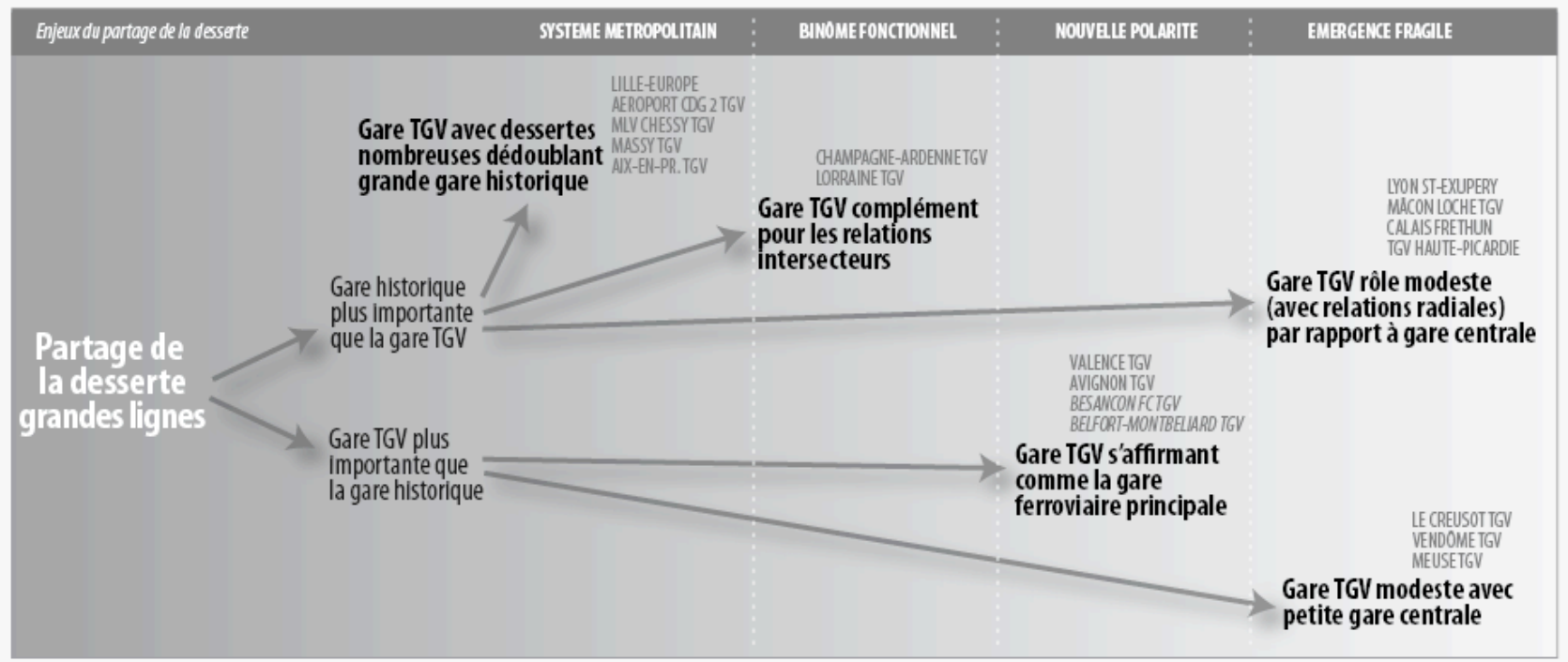

\section{2-1/ Des gares insérées dans un système métropolitain}

La première classe regroupe des gares TGV bénéficiant de capacités relationnelles fortes insérées dans des systèmes métropolitains (Lille-Europe, Massy TGV, Aéroport CDG 2 TGV, MLV-Chessy TGV, Aix-en-Pr. TGV). Ces gares n'en restent pas moins des nœuds complémentaires à de grandes gares centrales qui profitent au maximum de la mise en service d'infrastructures ferroviaires à grande vitesse. Ce dédoublement de la desserte ferroviaire entre les nœuds historiques en position de terminus et les nouvelles gares du réseau LGV assure une accessibilité de premier ordre lorsqu'elle est valorisée par une connexion efficace.

Le partage fonctionnel se fonde ici sur une répartition selon le type de flux : la gare TGV est desservie principalement ou exclusivement par des liaisons TGV interrégionales et/ou européennes tandis que la gare centrale capte les flux radiaux et constitue une centralité ferroviaire à l'échelle métropolitaine, régionale et nationale. Si cette complémentarité est évidente pour les couples de gares franciliennes, elle est aussi avérée dans le cas lillois. Toutefois, l'évolution de la répartition des dessertes TGV entre Lille-Flandres et Lille-Europe tend à estomper la spécialisation fonctionnelle des gares. La gare terminus dispose désormais de relations interrégionales tandis que la gare de Lille-Europe assure aussi des liaisons radiales (Menerault, 
2010). Désormais Lille-Flandres et Lille-Europe avec les TER-GV, disposent chacune de relations régionales, nationales et internationales.

Au sein de l'aire métropolitaine marseillaise, la complémentarité entre Aix-en-Provence TGV et Marseille St-Charles est davantage géographique que fonctionnelle : les deux gares bénéficient d'une desserte TGV comparable mais elles constituent deux points d'accès différents au réseau ferroviaire à grande vitesse. La gare TGV d'Aix-en-Provence, malgré l'abondance de l'offre, s'affirme plutôt comme un pôle relais destiné à offrir un accès complémentaire au réseau à grande vitesse afin de délester d'une partie de son trafic la gare centrale de Marseille St-Charles.

\section{2-2/ La constitution d'un binôme fonctionnel de gares TGV}

En assurant principalement l'accès aux liaisons à grande vitesse interrégionales, la gare TGV constitue une desserte complémentaire à la gare centrale desservie par les relations radiales. Les nœuds ferroviaires historiques bénéficiant d'une desserte TGV vers Paris par l'intermédiaire d'un raccordement avec le réseau LGV se retrouvent renforcés. Cependant, leur position nodale sur le réseau grande ligne est fragilisée par l'arrivée de la gare TGV accueillant les TGV « intersecteurs " qui transforme les gares centrales, jadis stations intermédiaires du réseau grande ligne, en « station terminus » pour les liaisons radiales.

Cette situation correspond principalement à la logique de desserte arborescente adoptée pour le TGV-Est. Les gares Champagne-Ardenne TGV et Lorraine TGV s'inscrivent dans des configurations spatiales originales fondées sur un partage fonctionnel complémentaire avec les gares du réseau ferroviaire classique. La constitution de barreaux de raccordement entre la ligne nouvelle à grande vitesse et le réseau classique permet aux gares centrales de Reims, Nancy et Metz de conserver leur fonction de desserte régionale et nationale en accueillant plus spécifiquement les flux radiaux à grande vitesse vers la capitale. Les gares TGV, desservies par des flux interrégionaux, constituent des haltes modestes par rapports aux gares historiques dont la fréquentation a été peu affectée par la mise en service de nouvelles gares. L'exemple de Reims montre bien que la nouvelle gare n'a pas fragilisé la fréquentation de la gare historique dont le rythme de croissance s'est accéléré avec la mise en service de la LGV Est.

\section{2-3/ Des gares TGV s'affirmant comme de nouvelles polarités ferroviaires}

Ce troisième type de partage fonctionnel s'effectue nettement en faveur de la gare TGV qui accueille l'essentiel du trafic TGV (radial et interrégional) de l'aire urbaine considérée. La gare nouvelle positionnée sur le réseau LGV s'impose comme le principal nœud ferroviaire pour les relations nationales, ce qui impacte plus ou moins fortement la dynamique de la gare historique qui se retrouve déclassée dans la hiérarchie ferroviaire. Le transfert des relations nationales vers la nouvelle gare a provoqué une baisse de leur fréquentation même si les gares du réseau classique continuent d'accueillir les liaisons grande ligne par corail ainsi que quelques TGV. Ces flux nationaux ont toutefois considérablement diminué et ces gares ont perdu la fonction de correspondance qu'elles jouaient entre flux régionaux et flux nationaux.

A Valence comme en Avignon, le partage fonctionnel s'est opéré en faveur des gares TGV, reliées à haute fréquence à des destinations variées, au détriment des gares centrales dont le trafic a fortement chuté. Desservies par quelques relations TGV radiales qui empruntent la ligne PLM pour desservir les petites villes intermédiaires de la vallée du Rhône, ces gares historiques ont assisté, avec la mise en place de la LGV Méditerranée à une chute brutale de leur fréquentation en raison du transfert de la plupart des relations nationales vers la nouvelle gare et de la disparition de la fonction de correspondance qu'elles remplissaient entre flux régionaux et flux à longue distance. Le renforcement de leurs dessertes locale et régionale leur a certes permis d'enregistrer une nouvelle progression de trafic, mais elles n'ont pas retrouvé le niveau de fréquentation qu'elles avaient avant la mise en service de la nouvelle ligne (Facchinetti-Mannone, 2002). Les gares TGV voient transiter désormais $70 \%$ du trafic ferroviaire des agglomérations, alors que les gares centrales accueillent majoritairement des flux de type pendulaire de portée plus réduite. Ces évolutions ont eu des conséquences sur les services et commerces des gares historiques et de 
leurs abords qui ont assisté à une baisse de leur chiffre d'affaires et pour certains d'entre eux à une fermeture de l'activité.

On peut rapprocher ces situations du cas de Besançon-Viotte qui subira, au moment de la mise en service du TGV Rhin-Rhône en décembre 2011, un affaiblissement de ses relations nationales au profit de la future gare de Besançon-Franche-Comté TGV. D'une quinzaine de relations quotidiennes cumulées (TGV et Corail) vers Paris, Lyon et Strasbourg, la gare de Viotte n'accueillera plus, en 2011, que 5 TGV vers ces mêmes destinations (4 TGV vers Paris et 1 vers Lyon et Strasbourg). Avec le transfert de la desserte TGV vers la nouvelle gare de BesançonFranche-Comté TGV (8 allers-retours quotidiens vers Paris, 5 vers Lyon et 5 vers Strasbourg), on peut faire l'hypothèse que le centre de gravité des relations ferroviaires à longue distance va changer de site.

\section{$\underline{\text { 2-4/ Des interactions limitées pour les gares faiblement desservies }}$}

Cette dernière classe se distingue par des relations ferroviaires relativement faibles dans les gares TGV. A l'exception notable de la gare TGV Haute-Picardie (voir ci-après), la médiocre desserte de cette catégorie est essentiellement radiale. Certaines d'entre-elles ont un rôle modeste par rapport à la gare centrale, nœud important pour les relations régionales (Mâcon, Calais) ou centralité ferroviaire majeure (Lyon). D'autres gares TGV, insérées au cœur d'espaces de faible densité, apportent une desserte à longue distance qui n'existait pas avant l'arrivée de la LGV (Le Creusot/ Montceau-les-M., Vendôme, Bar-le-Duc/ Verdun). Dans ces configurations, quelle que soit l'importance de la gare centrale, les relations inter-gares relèvent surtout de l'indifférence. II s'agit de nœuds d'échelles juxtaposées marqués par l'absence de liens entre les dynamiques de la gare centrale et de la gare sur LGV.

Les gares TGV aménagées en périphérie des villes petites et moyennes assurent, avec des fréquences et une offre de destinations variable selon la taille des pôles desservis, une fonction de desserte sur les grandes relations nationales. Les gares centrales des agglomérations à l'écart desquelles elles sont aménagées ont quant à elles une fonction de desserte locale et régionale. Gare TGV et gare historique constituent ainsi deux pôles aux fonctions bien distinctes, inscrits dans des réseaux de desserte d'échelles différentes. L'impact du dédoublement de la fonction ferroviaire a été dans ce cas plus ou moins marqué selon la position et la fonction qu'occupait la gare au sein du réseau classique.

Pour les villes situées sur un axe ferroviaire secondaire médiocrement équipé, les gares TGV ont très nettement amélioré l'accessibilité ferroviaire sans trop affecter les fonctions et la fréquentation des gares centrales. Ces dernières ont certes perdu des voyageurs et ont vu leur fonction de rabattement disparaître, puisque le trafic qui transitait par ces gares pour rejoindre le réseau principal y accède désormais directement par l'intermédiaire des nouvelles gares, dont le trafic s'accroît ; le rapprochement avec la capitale ayant provoqué une forte progression des déplacements pendulaires et professionnels. Le déclin de la fréquentation des gares historiques, nœuds secondaires assurant des liaisons locales et régionales vers un nombre de destinations réduit, s'est traduit par une diminution des fréquences de desserte. A Vendôme, la majeure partie des relations régionales est aujourd'hui assurée par autocars. Le cas de Mâcon est un peu atypique puisque la gare conventionnelle, fragilisée par le transfert des liaisons avec Paris vers la gare TGV de Mâcon-Loché, reste la plus fréquentée (elle concentrait en 2006, 70 \% du trafic ferroviaire de l'agglomération) grâce notamment au renforcement de sa fonction régionale sur l'axe Dijon-Lyon.

Le partage de la desserte apparaît dépendant de la position et de la taille des aires urbaines : une ville moyenne en position intermédiaire a peu de chance de bénéficier d'une desserte très étoffée. Cependant, il ne faut pas négliger l'importance des opportunités techniques offertes par l'infrastructure et en particulier les raccordements entre LGV et lignes classiques. L'existence d'une connexion ferroviaire peut apparaître discriminante dans le choix du partage de la desserte TGV : la constitution d'un binôme fonctionnel dans le cas des gares du TGV-Est est permise par les raccordements ferroviaires opérés. Dans d'autres cas, comme dans l'aire urbaine de Belfort et 
Montbéliard, le croisement avec le réseau classique (axe Besançon-Montbéliard-Belfort-Mulhouse en double voie électrifiée) n'a pas donné lieu à une interconnexion. Ainsi, les gares centrales de Montbéliard et de Belfort ne seront plus desservies ni par des TGV ni par les liaisons grandes lignes. La situation est comparable dans le cas d'Amiens, écartée du tracé du TGV-Nord, et de StQuentin. Le positionnement de la gare TGV Haute-Picardie et l'absence de connexion avec la médiocre voie ferroviaire (non électrifiée) reliant les deux villes n'a finalement pas permis d'assurer la complémentarité des gares. La ville d'Amiens qui est l'une des rares capitales régionales à ne pas être accessible en TGV, s'intéresse d'ailleurs aujourd'hui à un raccordement à proximité de l'aéroport de Roissy CDG pour bénéficier de liaisons TGV interrégionales, ce qui reviendrait, en somme, à rendre en partie caduque la desserte et donc l'existence même de la gare TGV HautePicardie.

Quelle que soit la modification de la desserte ferroviaire des gares centrales imposée par de nouvelles implantations périphériques, la performance de relations intergares, étudiée dans la partie suivante, apparaît comme un enjeu clé pour compenser l'éloignement de la gare, accéder à une desserte à grande vitesse ou constituer un système de transport métropolitain intégré.

\section{3/ Les performances variées de la connexion intergare : l'accessibilité à la gare TGV en question}

\section{3-1/ L'accès en transport collectif aux gares TGV : un enjeu majeur traditionnellement négligé}

Pour les collectivités, la problématique de l'accès à la gare TGV est décisive car elle conditionne l'intégration territoriale de la gare et donc sa capacité à interagir avec la dynamique urbaine. Le contexte géopolitique de l'aménagement du territoire est marqué par une montée en puissance des collectivités locales et structures intercommunales. Depuis le projet de LGV Est (2007), ces dernières participent à hauteur de $30 \%$ au financement de la nouvelle infrastructure alors que la construction des Lignes à Grande Vitesse était jadis exclusivement du ressort de l'Etat. Dans le cas de la LGV Rhin-Rhône, les communautés d'agglomérations (Besançon, Montbéliard, Belfort, Dijon, Mulhouse et Strasbourg), les conseils généraux (Doubs, Territoire de Belfort, Haute-Saône, Haut-Rhin, Bas-Rhin et Côte-d'Or) et les conseils régionaux (Franche-Comté, Alsace et Bourgogne) apportent $650 \mathrm{M} €$ au projet sur un total de plus de 2 milliards d' $€$. Cette situation donne de l'importance aux acteurs locaux qui apparaissent directement concernés par la nouvelle liaison ferroviaire, la localisation des gares et la répartition des dessertes.

Or, si les acteurs locaux ne disposent que d'un rôle modeste sur les choix de localisation, ils ont la charge de l'organisation des réseaux de transport pour rendre accessible la nouvelle gare TGV. La mise en place d'une intermodalité performante sera d'autant plus importante que les nouvelles gares TGV polarisent un espace qui dépasse l'échelle de l'agglomération sans avoir une fonction de desserte locale et régionale en transport en commun. Les acteurs locaux cherchent ainsi à pallier l'éloignement des gares nouvelles par la mise en place de liaisons performantes entre les plates-formes centrale et périphérique. L'objectif étant d'assurer les interdépendances entre deux dessertes ferroviaires spatialement dissociées et de favoriser ainsi le co-développement d'une accessibilité locale, régionale et (inter)nationale par les transports collectifs. L'existence d'un " trinôme d'interconnexion » ferroviaire, pour faire le parallèle avec le concept développé par Jean Varlet à propos de l'intégration territoriale des plates formes aéroportuaires, serait en mesure d'affirmer le rôle de plaque tournante des gares TGV et de réduire leur isolement vis-à-vis de la plupart des fonctions urbaines.

Malgré l'importance de ces enjeux, la localisation des gares est traditionnellement marquée par une faible prise en compte de la desserte en transports collectifs. En France, si l'on excepte les navettes ferroviaires spécifiques (Lyon St-Exupéry, Champagne-Ardenne TGV et, en 2011, Besançon FC TGV), seules les gares de Valence TGV et de Calais Frethun sont positionnées au 
croisement d'une ligne TER. Les possibilités de connexion des gares TGV au réseau classique n'ont été que tardivement prises en compte, même lorsqu'elles étaient techniquement réalisables.

Dans plusieurs cas, en effet, le positionnement de la gare néglige la proximité de la desserte ferroviaire existante. A Vendôme, la nouvelle gare se trouve à $5 \mathrm{~km}$ au nord du croisement entre la LGV Atlantique et la ligne classique à voie unique non électrifiée qui relie Vendôme à Troo. La proximité de cette infrastructure, aujourd'hui exploitée pour le transport de fret céréalier et dans un cadre touristique, n'a pas été prise en compte lors de l'implantation de la gare. La gare TGV du Creusot a été aménagée à quelques centaines de mètres de la petite gare de Montchanin, à laquelle elle est reliée par une voie non électrifiée utilisée pour la maintenance de la LGV. La modernisation de cette infrastructure, aujourd'hui en cours d'étude, aurait permis de prolonger certains des TER circulant sur le réseau classique vers la gare TGV. Dans le contexte de déclin du transport ferré régional de voyageurs qui a accompagné la mise en service des premières gares TGV, la modernisation des lignes conventionnelles imposait des investissements jugés trop lourds par rapport au surcroît de trafic attendu (Facchinetti-Mannone, 2006). Ces contraintes financières continuent de peser lourdement sur les choix de localisation de gares. En région Lorraine, la gare TGV a été implantée à Louvigny, contre l'avis de la FNAUT et de plusieurs collectivités locales, à quelques kilomètres du croisement de la LGV avec la ligne classique du sillon mosellan (site de Vandières).

Au cours des années 90 , les études d'implantation de gares intègrent progressivement ces opportunités de connexion au réseau classique, même si le contexte ou des contraintes techniques et financières amènent à les rejeter (Haute Picardie TGV) ou à les reporter. En Avignon, les possibilités de raccordement à la ligne classique ont été un élément prépondérant dans le choix du site de la gare TGV. La proximité de l'agglomération était propice à la création d'un lien inter-gares. Le site de Saint-Gabriel, privilégié par la SNCF, permettait d'optimiser la connexion. II offrait au TGV la possibilité de desservir les deux gares en bénéficiant de correspondances quais à quais avec le TER. Le projet, qui menaçait les exploitations maraichères de la ceinture verte, suscita une vive opposition locale et fut abandonné au profit d'une gare TGV à Courtine. Le site retenu permet de raccorder la nouvelle gare à la ligne PLM, par le biais d'un tronçon à voie unique banalisée de $1300 \mathrm{~m}$, mais offre des conditions de correspondances moins favorables, les liaisons TER en provenance ou à destination du sud imposant un rebroussement en gare centrale. Les terrains nécessaires à la réalisation de la "virgule " ont fait l'objet d'une réserve d'emprise, mais le projet a été reporté à plusieurs reprises faute d'un accord sur son financement.

L'intégration des gares TGV aux réseaux de transports collectifs urbains s'est aussi avérée timide. La localisation des gares au sein des Périmètres de Transports Urbains des agglomérations est un élément nécessaire mais non suffisant à une insertion dans les réseaux de transports urbains. Si les gares périphériques implantées en limite des PTU sont le plus souvent desservies par prolongement de lignes existantes, cette intégration n'est pas toujours systématique. La gare TGV d'Aix en Provence, en limite du territoire communal n'est pas desservie par le réseau de bus urbain. A Mâcon, la gare TGV n'a été que récemment intégrée au réseau de transport urbain : la ligne 12 relie le centre à l'Espace Entreprises Macon-Loché, aménagé à proximité de la gare TGV, mais jusqu'en 2009, son terminus se trouve à $400 \mathrm{~m}$ de la gare !

\section{3-2/ La diversité des liens entre gare historique et gare sur LGV}

L'évaluation de la performance des liaisons entre les couples de gares analysés repose sur plusieurs critères : elle intègre le(s) type(s) de mode utilisé, la nature des dessertes mises en place (dessertes spécifiques à la gare TGV ou adaptation de services pré-existants) ainsi que la performance des relations analysée en fonction de la rapidité des liaisons, de leur fréquence et du degré d'intégration horaire et tarifaire entre offre de rabattement et desserte TGV. La typologie ainsi obtenue (voir figure 5) souligne la variété des performances de ces liaisons inter-gares susceptibles de renforcer l'intégration territoriale des nouvelles gares TGV. 
Figure 5: Typologie des liaisons intergares en transport collectif

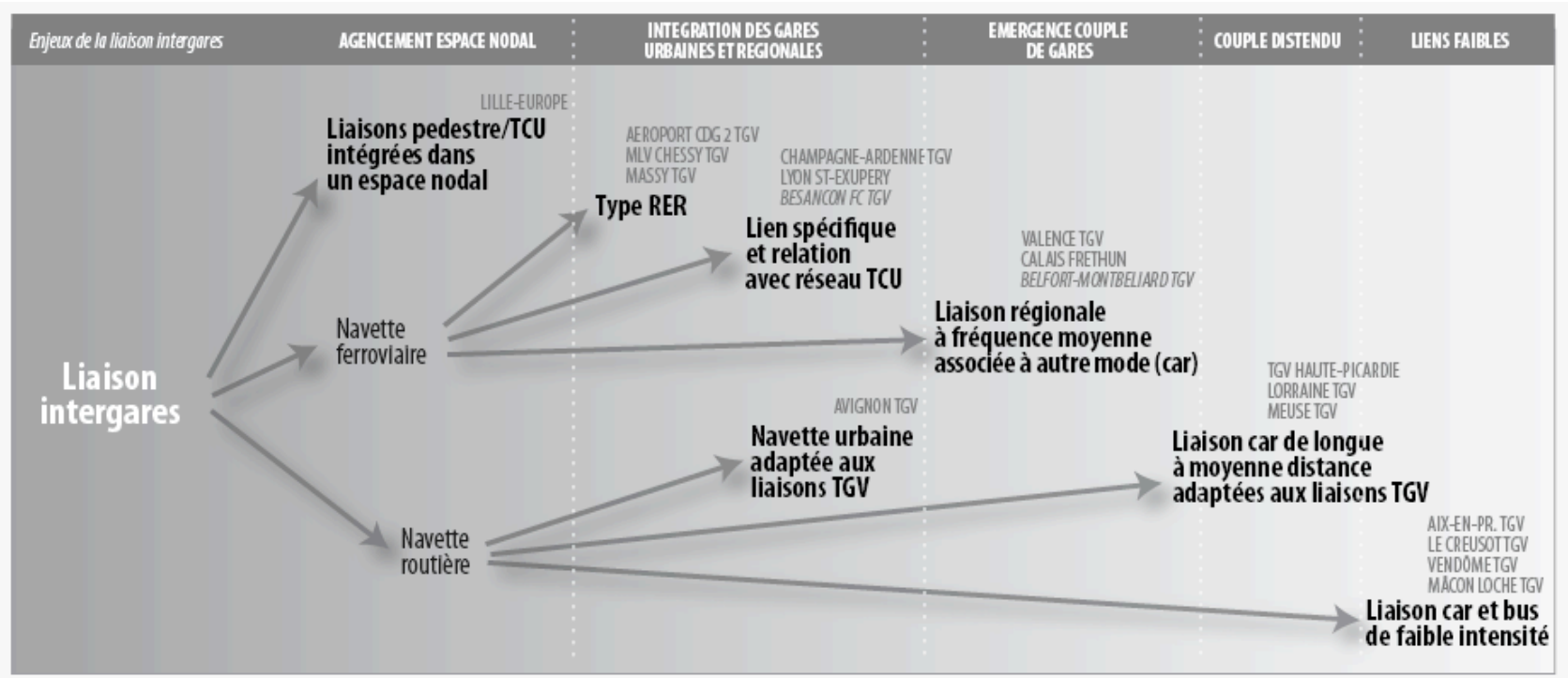

\section{3-2-1/ L'agencement d'un espace nodal}

Cas unique, l'insertion de la gare de Lille-Europe à quelques centaines de mètres de la gare de Lille-Flandres créée les conditions d'une interaction forte entre les deux nœuds ferroviaires. Après avoir recherché la connexion par les modes de transports collectifs urbains en site propre (MétroVal et Tramway détourné pour la desserte de Lille-Europe), l'attention se porte aujourd'hui davantage sur l'amélioration de la continuité urbaine entre deux sites, distants de quelques centaines de mètres, pour faciliter les cheminements pédestres intergares (par exemple, par la mise en place d'une signalétique adaptée). Lille-Europe et Lille-Flandres apparaissent ainsi comme deux éléments d'un même espace nodal.

\section{3-2-2/ L'intégration des gares TGV dans un réseau de transport urbain ou régional}

Les liaisons intergares peuvent être motivées par l'intégration d'une nouvelle gare ferroviaire dans le fonctionnement urbain. Cette ambition est facilitée par un positionnement de la gare en limite d'agglomération dans le périmètre de l'AOTU. Par exemple, la gare de Champagne-Ardenne TGV en bordure de l'agglomération rémoise et déjà desservie par une navette ferroviaire, va prochainement être intégrée au réseau de tramway (mise en service le 11 avril 2011). Avec leurs singularités, le cas des gares sur LGV en Île-de-France interconnectées avec le RER, celles desservies par des navettes ferroviaires spécifiques comme à Lyon (hors-PTU) ou par une navette bus dédiée comme en Avignon (et non par un bus urbain régulier), entrent dans cette catégorie. Reposant sur des liaisons directes à haute fréquence et/ou une intégration horaire et tarifaire complète, ce type de lien favorise, quel que soit le mode utilisé, les conditions d'une intermodalité performante apte à compenser l'éloignement des centres urbains. Cette intégration de toutes les échelles de mobilité de l'agglomération garantit en outre un fonctionnement plus équilibré entre les deux gares. Cependant, l'absence d'intégration tarifaire et le coût élevé des navettes (exemple de RhônExpress entre Lyon Part-Dieu et Lyon St-Exupéry) limitent dans certains cas, l'intérêt de la liaison pour l'usager.

\section{3-2-3/ L'émergence de couple de gares bénéficiant d'un potentiel d'interconnexion}

Ce troisième type désigne des liaisons plus distantes, généralement liées à une localisation de la gare TGV hors PTU ou en bordure, et qui, malgré un niveau de performance correct, ne permettent pas une valorisation optimale de l'intermodalité. Dans certains cas, des contraintes d'exploitation de nature technique (l'optimisation des correspondances entre TGV et TER se révèle plus complexe lorsque la gare est implantée, comme à Valence ou à Calais, à l'intersection de la LGV et de la ligne classique) ou financière (Besançon) ne permettent pas aux navettes ferroviaires 
d'assurer toutes les correspondances. L'offre de rabattement est alors complétée par des navettes routières interurbaines, dont les performances, en termes de temps d'accès notamment, sont moindres.

\section{3-2-4/ Des couples de gares distendus par la longueur des rabattements}

La spécificité de ce type de liaisons intergares résulte du mode routier, du caractère spécifique de la navette et de la plus ou moins grande distance qui sépare la gare centrale de la gare TGV. Ces liaisons routières interurbaines, généralement organisées par les conseils généraux, visent exclusivement à desservir les nouvelles gares exurbanisées par la mise en place de navettes offrant des correspondances horaires de bon niveau: il y a généralement toujours un car dans la demi-heure précédant ou suivant le départ ou l'arrivée du TGV. Seulement, la qualité de la desserte est fragilisée par la faible fréquence des navettes, par le risque de majoration des temps de trajet en fonction de la congestion routière, ainsi que l'absence de desserte d'autres sites urbains.

De plus, l'éloignement des nouvelles gares apparaît globalement préjudiciable au déplacement de l'usager et au gain de temps offert par la performance du système TGV. Par exemple, pour un trajet entre Amiens (gare) et Aix-en-Provence (gare routière), un tiers du temps total de trajet est consacré au pré et post acheminement et ce, malgré des correspondances efficaces en gare TGV (Sur un temps total de 5h30, le trajet en TGV est d'environ 4h). Ainsi les gains de temps qu'offre le TGV sont souvent amoindris par les temps d'accès aux gares TGV périphériques.

\section{3-2-5/ Des liens faibles ou inexistants par les transports collectifs}

Ce dernier groupe rassemble des dessertes intergares de qualité variable réalisées par des navettes routières peu performantes. En particulier, la segmentation de l'offre urbaine et interurbaine d'accès à la gare TGV participe à la faible visibilité de la desserte en transports collectifs. A Mâcon, par exemple, la perte de la fonction de correspondance de la gare centrale n'a pas été compensée par la mise en place d'une offre de rabattement performante vers la gare TGV, la taille de l'agglomération ne permettant pas d'assurer une rentabilité suffisante à ce type de service ; et en définitive, les deux gares restent faiblement connectées. A Vendôme, les deux gares se juxtaposent de façon totalement indépendante, comme l'atteste les faibles performances des navettes bus qui les relient. Ces dernières ont d'ailleurs davantage pour fonction de relier la gare TGV au centre ville et non de constituer une connexion avec la gare historique. Dans ces espaces de faible densité, la précarité des relations entre gare centrale et gare sur LGV se traduit par une intermodalité déficiente qui a pour corollaire une forte pression automobile aux abords de la station. La saturation des zones de stationnement n'est toutefois pas spécifique à cette catégorie de gares sur LGV même si elle s'avère peut-être plus critique qu'ailleurs.

\section{3-3/ Perspectives et débats : vers une meilleure prise en compte de la liaison intergare ?}

A première vue, l'enjeu de la desserte des gares TGV semble de mieux en mieux identifié à défaut de remettre en cause leur modèle de localisation. Dans les années 1980 et 1990, les gares nouvelles des LGV Sud-Est, Atlantique et Nord n'ont pas fait l'objet d'une réflexion très approfondie sur les perspectives de desserte en transport collectif. Lille-Europe mis à part, la localisation de ces nouvelles gares est davantage dictée par les contraintes techniques imposées par les choix de tracé. Le positionnement des nouvelles haltes à proximité des lignes classiques répond à des problématiques de maintenance ou de connectivité du réseau en cas de problèmes sur la LGV mais n'a pas été perçu comme un enjeu pour la desserte intergare.

Pour les LGV Méditerrannée (2001) ou LGV Est (2007), les différents acteurs apparaissent plus soucieux de limiter l'éloignement des nouvelles gares et d'offrir une réelle alternative à l'automobile pour l'accès au TGV. Cette mobilisation se traduit par des réflexions plus étroites sur la localisation des gares TGV en fonction des opportunités de desserte ferroviaire. Le résultats est cependant mitigé puisque, si deux gares TGV ont profité de ces nouvelles considérations (Valence TGV et Champagne-Ardennes TGV), trois autres n'ont pas encore concrétisé des potentialités pourtant 
envisagées : la navette ferroviaire de la gares TGV d'Avignon, intégrée à la réouverture de la ligne Avignon-Carpentras, n'est pas encore en service, la connexion de Lorraine TGV au réseau classique dépend de la reconstruction de la gare sur un autre site tandis que le projet de TCSP envisagé à Aix-en-Provence TGV apparaît désormais hypothétique.

A l'heure actuelle, le renouveau des déplacements ferroviaires à courte et moyenne distance, impulsé par la régionalisation, et le contexte d'éco-mobilité plaident en faveur de l'intégration des nouvelles gares au réseau classique. D'autant que la participation croissante des collectivités locales au financement des nouvelles LGV explique l'intérêt qu'elles portent à ces connexions qui permettent d'atténuer l'éloignement des gares périphériques et les conséquences d'un partage fonctionnel déséquilibré.

Si l'amélioration des connexions des gares TGV existantes au réseau conventionnel, en cours de réflexion sur plusieurs sites, est parfois compliquée, la création de nouvelles gares devrait permettre d'intégrer plus systématiquement les enjeux de desserte ferroviaire pour constituer des pôles intermodaux performants. L'exemple du projet de TGV Rhin-Rhône, qui sera mis en service fin 2011, est à ce titre doublement instructif : d'une part, parce que la connexion des gares TGV au réseau classique n'a pas été complètement anticipée et d'autre part, parce qu'il montre que des problématiques similaires donnent lieu à des interventions différentes.

Le projet de TGV Rhin-Rhône prévoit la création de deux gares nouvelles intermédiaires sur le tracé de la branche Est : l'une en périphérie de Besançon pour desservie l'aire urbaine de la capitale régionale (220 000 habitants), l'autre au cœur de l'aire urbaine multipolaire de Belfort et Montbéliard (300 000 habitants environ). Même si ces deux gares seront intégrées au réseau TER, il est difficile de conclure à une meilleure prise en compte de l'accès aux gares TGV. La nouvelle gare de Besançon Franche-Comté TGV doit sa navette ferroviaire à une opportunité technique résultant du besoin de connecter la LGV au réseau classique pour offrir des itinéraires de délestage. La réouverture de la ligne ferroviaire Belfort-Delle permettra, quant à elle, de relier la nouvelle gare TGV à Belfort mais pas à Montbéliard. Or, la prise en compte des enjeux de rabattement aurait dû concentrer l'attention sur le croisement entre la LGV et le réseau classique pour utiliser les relations TER existantes entre Montbéliard et Belfort pour la desserte de la gare TGV.

En outre, alors qu'aucun des sites accueillant les nouvelles gares du TGV Rhin-Rhône ne bénéficiait de desserte en transport collectif, la liaison avec les trois gares centrales concernées fait l'objet de trois interventions différentes: Entre la gare Besançon-Viotte et la gare TGV, le raccordement ferroviaire, d'origine technique, est inclus dans le financement global de la LGV; Entre la gare historique de Belfort et la gare TGV, la réouverture de la ligne ferroviaire BelfortDelle, plus longue et plus coûteuse pour les collectivités, est inscrite au contrat de projet Étatrégion 2007-2013 (Participation de l'Etat de l'ordre de 25\%) ; Enfin, entre Montbéliard et la même gare TGV, la navette routière sera à la charge de l'agglomération et aucune participation de l'État n'est à attendre.

\section{Conclusion}

L'intégration territoriale des gares TGV varie ainsi selon la localisation des nouvelles gares TGV au sein des aires urbaines qu'elles desservent, du nouveau partage de la desserte ferroviaire entre gare sur LGV et gare sur réseau classique et de la qualité des liaisons intergares. Plusieurs grandes tendances se dégagent de l'analyse typologique menée en fonction de chacun de ces critères :

En termes de localisation, l'optimisation du fonctionnement du système français à grande vitesse conduit à privilégier des gares TGV excentrées, en périphérie d'un ou plusieurs pôles urbains. L'implantation des gares en bordure de l'espace aggloméré (Avignon, Reims), garante d'une bonne intégration territoriale, est finalement assez rare et les localisations au cœur des villes, favorables à une densification de la centralité, restent 
exceptionnelles en raison des difficultés posées par l'insertion des LGV en tissu urbain dense. Le cas unique de Lille-Europe résulte d'ailleurs d'une forte mobilisation des collectivités locales et des opportunités foncières liées aux anciennes fortifications de la zone non aedificandi (Menerault, 2009).

- En termes de partage fonctionnel, l'impact du dédoublement de la desserte ferroviaire a été plus ou moins marqué selon la position et la fonction qu'occupait la gare historique au sein du réseau classique. Les gares centrales confortées par l'arrivée du TGV, malgré la mise en service d'une ou plusieurs gares nouvelles, sont le reflet de l'émergence de systèmes ferroviaires métropolitains fondés sur une complémentarité des gares. Par contre, en se substituant aux gares centrales pour la desserte inter-régionale et nationale, les nouvelles gares sont à l'origine de déséquilibres susceptibles de modifier, en fonction du degré de déclassement des gares centrales, les structures et dynamiques spatiales des aires urbaines desservies.

- En termes de liaisons inter-gares, l'implantation des nouveaux nœuds ferroviaires dans les périmètres de transport urbain ou sur des sites offrant des opportunités de connexion au réseau classique, rend possible des liens plus étroits; qu'ils soient activés (Reims, Valence, gares franciliennes, Lille), en cours d'activation (Avignon) ou sous valorisés (Aix, Creusot, Vendôme, Mâcon ....). L'insertion au réseau routier interurbain ou au réseau ferroviaire régional assure une intégration territoriale très variable, et l'interconnexion reste souvent marquée par un temps de trajet pénalisant qui nécessite à minima une optimisation des correspondances.

Le croisement des enjeux mis en évidence par cette triple approche typologique (voir figure 6) souligne la grande diversité du degré d'intégration territoriale des gares TGV. Ces différents degrés d'intégration territoriale sont le résultat de jeux d'acteurs divers et complexes, reflets des concurrences ou complémentarités entre collectivités territoriales de différents niveaux, gestionnaires d'infrastructure et opérateurs ferroviaires. La typologie proposée, même si elle ne préjuge en rien du potentiel de développement urbain et économique des sites ferroviaires, conduit à interroger les stratégies de valorisation mises en œuvre par les collectivités autour de gares présentant des degrés d'intégration territoriale très dissemblables.

Figure 6 : Croisement des enjeux concernant la localisation, la desserte et les liaisons intergares

\begin{tabular}{|c|c|c|c|c|}
\hline $\begin{array}{l}\text { Degré } \\
\text { " d'intégration } \\
\text { territoriale » } \\
\text { des gares TGV }\end{array}$ & $\begin{array}{l}\text { (1) } \\
\text { Localisation }\end{array}$ & $\begin{array}{l}(2) \\
\text { Partage } \\
\text { fonctionnel }\end{array}$ & $\begin{array}{l}(3) \\
\text { Liaison } \\
\text { intergares }\end{array}$ & Gares TGV \\
\hline Intégration & $\begin{array}{l}\text { Contact centralité } \\
\text { métropolitaine }\end{array}$ & $\begin{array}{l}\text { Système } \\
\text { métropolitain }\end{array}$ & $\begin{array}{l}\text { Agencement } \\
\text { espace nodal }\end{array}$ & Lille Europe \\
\hline Synergie & $\begin{array}{l}\text { Desserte fonction } \\
\text { métropolitaine } \\
\text { Continuité } \\
\text { organisation } \\
\text { urbaine }\end{array}$ & $\begin{array}{l}\text { Système } \\
\text { métropolitain } \\
\text { Binôme } \\
\text { fonctionnel }\end{array}$ & $\begin{array}{l}\text { Intégration des } \\
\text { gares urbaines et } \\
\text { régionales }\end{array}$ & $\begin{array}{l}\text { Aéroport CDG } 2 \text { TGV } \\
\text { Marne-la-Vallée Chessy } \\
\text { TGV } \\
\text { Champagne-Ardennes TGV } \\
\text { Avignon TGV } \\
\text { Massy TGV } \\
\text { Lyon Saint-Exupéry TGV }\end{array}$ \\
\hline Cohabitation & $\begin{array}{l}\text { Accompagnement } \\
\text { dilution des } \\
\text { régions urbaines }\end{array}$ & $\begin{array}{l}\text { Binôme } \\
\text { fonctionnel } \\
\text { Nouvelle polarité }\end{array}$ & $\begin{array}{l}\text { Emergence } \\
\text { couple de gares } \\
\text { Couple distendu }\end{array}$ & $\begin{array}{l}\text { Aix-en-Provence TGV } \\
\text { Valence TGV } \\
\text { Lorraine TGV } \\
\text { Besançon Franche-Comté } \\
\text { TGV } \\
\text { Belfort-Montbéliard TGV }\end{array}$ \\
\hline Indifférence & $\begin{array}{l}\text { Equipement ex- } \\
\text { urbanisé }\end{array}$ & $\begin{array}{l}\text { Emergence } \\
\text { fragile }\end{array}$ & Liens faibles & $\begin{array}{l}\text { Calais-Frethun TGV } \\
\text { Mâcon TGV } \\
\text { TGV Haute-Picardie } \\
\text { Le Creusot TGV } \\
\text { Meuse TGV } \\
\text { Vendôme TGV }\end{array}$ \\
\hline
\end{tabular}

- L'intégration n'est, en définitive, effective que dans le cas unique de la gare TGV de LilleEurope. La nouvelle desserte sur le réseau à grande vitesse participe à la densification 
d'un espace nodal qui repose sur des liaisons potentiellement complémentaires (Paris, Londres, Bruxelles, TER-GV) et la constitution d'une accessibilité métropolitaine multiniveaux.

- La synergie entre gare TGV et gare centrale existe lorsqu'un couple de gares a des interactions étroites, potentiellement structurantes, soit par l'insertion spatiale à l'aire urbaine, soit par l'insertion fonctionnelle au réseau de transport collectif urbain ou régional.

- Le terme de cohabitation renvoie à une coexistence technique des gares qui n'affirment pas d'interrelations marquées en raison d'une distance pénalisante, d'une superposition de la desserte et/ou d'une faiblesse des liens intergares. Les relations avec la gare TGV restent fragiles malgré un potentiel de complémentarité de desserte ou d'assimilation au fonctionnement urbain.

- Enfin l'indifférence pose la question de la localisation des gares qui apparaissent surimposées au maillage du réseau de transport et à l'organisation des aires urbaines à proximité.

Au-delà de l'approche typologique, les exemples analysés, et notamment le cas des gares nouvelles du TGV Rhin-Rhône, montrent qu'il y a encore du chemin à parcourir pour réellement valoriser l'intégration territoriale des gares TGV dans les projets de grande vitesse ferroviaire en France. Même si le positionnement de la gare ne préjuge en rien de sa fréquentation, ni de l'activation autour d'elle d'un potentiel de développement urbain ou économique, la relative faiblesse de la prise en compte de l'accessibilité s'avère pourtant problématique par rapport à la maîtrise des déplacements automobiles, à la consommation foncière en périphérie (souvent en décalage avec la planification urbaine) ou par rapport au temps de pré et post acheminement. L'éloignement des gares TGV couplé à la faiblesse des interconnexions avec le réseau classique peut également limiter, voir supprimer, toute opportunité de développement de la grande vitesse régionale. Par exemple, le modèle des TER-GV du Nord-Pas-de-Calais ne pourra pas être reproduit sous cette forme dans la métropole inter-régionale Rhin-Rhône (entre Dijon, Besançon, Belfort, Montbéliard et Mulhouse) faute de pouvoir coupler utilisation de la LGV et desserte des villes-centre.

Une des pistes qui pourrait être envisagée pour encourager la prise en compte de l'accessibilité des nouvelles gares en amont des projets de LGV serait un calcul global des coûts (LGV+Gares+Accès aux gares). Ainsi, le couplage du gain d'une desserte périphérique pourrait être mis en balance avec le coût d'une liaison ferroviaire intergare et la majoration des temps de pré et post acheminement. L'objectif recherché est une meilleure prise en compte globale du système de mobilité résultant de la mise en service d'une LGV. II peut en résulter soit une intégration des problématiques d'accès aux gares TGV dans le projet global, soit une considération nouvelle des opportunités de desserte des gares historiques qui bénéficient déjà d'une forte accessibilité urbaine et régionale. Ce cumul des coûts de l'intégration territoriale des gares TGV aurait pour avantage de justifier la participation des collectivités locales au financement de l'infrastructure ferroviaire à grande vitesse et de rechercher des solutions qui valorisent l'existant. 


\section{Bibliographie}

AUPHAN E. (1995), "La gare TGV Lorraine : un équipement inutile ? ", $120^{\text {ème }}$ congrès national des Sociétés Historiques et Scientifiques, Grands travaux, Aix en Provence, pp 2938.

AUPHAN E. (2002), "Le TGV Méditerranée: un pas décisif dans l'évolution du modèle français à grande vitesse » », in Revue Méditerranée $\mathrm{n}^{\circ}$ 1-2, pp. 19-26.

BARRE A. et MENERAULT P. (dir.) (2001), Gares et quartiers de gares : signes et marges. Lille, Rennes et expériences internationales (Italie, Japon, Pays-Bas), Actes du séminaire international du 22 mars 1999 à Villeneuve d'Ascq, Arcueil, INRETS, 216 p.

CHAMOIN P. (1994) « Interconnexion des gares nouvelles », R.G.C.F., juin/juillet 1994, pp. 135-141.

CHAPELON L., LECLERC R. (2007), Accessibilité ferroviaire des villes françaises en 2020, Reclus, Paris : La documentation française, $171 \mathrm{p}$.

CONSEIL ECONOMIQUE ET SOCIAL LORRAINE (2002), Les enjeux du TGV Est Européen et du positionnement de la gare d'interconnexion Lorraine, séance plénière du 17 mai 2002, $59 \mathrm{p}$.

EMANGARD P.H, BEAUCIRE F. (1985), « Du bon et du mauvais usage des gares TGV dans les régions traversées », Revue de Géographie de Lyon, vol. 60 n 4, pp. 359-374.

FACCHINETTI-MANNONE V. (1999), « Principes d'implantation et effets urbains des gares TGV en France: bilan de 17 ans d'aménagement ferroviaire », Annales des Ponts et Chaussées $\mathrm{n}^{\circ}$ 89, pp. 45-52.

FACCHINETTI-MANNONE V. (2002), « Dynamiques de l'accessibilité ferroviaire des villes desservies par le TGV Méditerranée et intégration territoriale des nouvelles gares TGV », Revue Méditerranée $\mathrm{n}^{\circ} 1-2$, «Systèmes de transport et mutation de l'espace méditerranéen » pp. 27-34.

FACCHINETTI-MANNONE V. (2006), "Gares ex-urbanisées et développement urbain : le cas des gares TGV bourguignonnes ", Revue de Géographie de l'Est T. XLVI, $\mathrm{n}^{\circ}$ 1-2, pp.15-23.

FACCHINETTI-MANNONE V. (2007), " La integracion territorial de las nuevas estaciones TAV perifericas: el caso frances », Cuadernos de Ingeniera y territorio 10, Acta de la Jornada Europea « Alta Velocidad y Territorio », Ciudad Real, pp. 73-91.

FACCHINETTI-MANNONE V., BAVOUX J-J. (2008), "Le problème de l'optimisation simultanée de la vitesse et de la desserte. Le choix de localisation des gares TGV », Contribution au Geopoint 2008 "Optimisation de l'espace géographique et satisfactions sociétales », pp 65-70.

FACCHINETTI-MANNONE V., RICHER C. (2008), "Gares centrales et gares TGV bis en France : l'enjeu du doublement de la fonction ferroviaire », III jornada europea sobre alta velocidad y territorio, Universidad de Castilla-la Mancha, Ciudad Real.

FACCHINETTI-MANNONE V., BAVOUX J-J. (2010), " L'implantation des gares TGV en France: tensions interscalaires, jeux d'acteurs et recompositions spatiales ", Belgéo, numéro spécial "Villes et grands équipements de transport: compétition, tensions, recompositions », n`1-2, pp. 9-22.

MENERAULT Ph. (2007), «Réseaux de transports publics à grande vitesse et aménagements, au croisement des échelles spatiales et temporelles ", in DUPUY G., GENEAU DE LAMARLIERE I., Nouvelles échelles des firmes et des réseaux. Un défi pour l'aménagement, L'Harmattan, pp. 91-120. 
MENERAULT Ph. (2008), " Sistemas polarizados regionales de ciudades y Alta Velocidad Regional en Francia. El caso de Nord-Pas-de-Calais », III jornada europea sobre alta velocidad y territorio, Universidad de Castilla-la Mancha, Ciudad Real.

MENERAULT Ph. (2009), " Gares ferroviaires et projets métropolitains », in Lille Métropole, laboratoire du renouveau urbain, Marseille, Ed. Parenthèses, pp. 101-122

MENERAULT Ph. (2010), "Grande vitesse et système métropolitain : une troisième gare TGV à Lille, enjeux et limites ", Communication au colloque "Gares et territoires de la grande vitesse ferroviaire », 16, 17, 18 juin 2010, Dijon et Besançon

OLLIVRO J. (1994), Essai de modélisation d'une implantation ferroviaire. L'exemple du TGV Méditerranée, Université de Haute-Bretagne, Rennes, 870 p.

PERIN P. (1999), «La gare TGV Haute-Picardie : six cents voyageurs par jour au milieu des betteraves ", Annales des Ponts et Chaussées, n89, pp. 32-38.

RICHER C., BERION P. (2010), « Le rôle des grandes infrastructures dans la structuration des espaces régionaux: le cas de l'arrivée du TGV dans le réseau métropolitain RhinRhône ", Belgéo, numéro spécial « Villes et grands équipements de transport : compétition, tensions, recompositions ", n¹-2, pp.159-170.

TROIN J.F. (1995), Rail et aménagement du territoire, Aix-en-Provence, Edisud, 264 p.

TROIN J.F. (1997), "Les gares TGV et le territoire: débat et enjeux. », Annales de Géographie n 593-594, pp.34-50.

TROIN J.F (1998) « Des gares TGV hors la ville : une aberration française ? », Urbanisme $\mathrm{n}^{\circ} 302$, pp 37-38.

TROIN J.F. (2008), Les gares nouvelles du TGV « exurbanisées ». Fonctionnement et relation au territoire, Rapport réalisé pour la DIACT, FNAUT, 66 p.

VARLET J. (1992), "Réseaux de transports rapides et interconnexions en Europe occidentale », L'information géographique Vol. 56, n³, pp. 101-114.

VARLET J. (2000), « Dynamique des interconnexions des réseaux de transports rapides en Europe : devenir et diffusion spatiale d'un concept géographique », Flux n41, pp. 5-16

ZEMBRI P. (1992), "TGV - réseau classique : des rendez vous manqués ? ", Transports Urbains $\mathrm{n}^{\circ} 75$, pp 21-24. 